\title{
GROUP-WISE ANALYSIS ON MYELINATION PROFILES OF CEREBRAL CORTEX USING THE SECOND EIGENVECTOR OF LAPLACE-BELTRAMI OPERATOR
}

\author{
Seung-Goo Kim ${ }^{a \star \star} \quad$ Johannes Stelzer ${ }^{a} \quad$ Pierre-Louis Bazin ${ }^{a}$ \\ Adrian Viehweger ${ }^{b} \quad$ Thomas Knösche $e^{a}$ \\ ${ }^{a}$ Max Planck Institute for Human Cognitive and Brain Sciences, Leipzig, Germany. \\ ${ }^{b}$ University of Leipzig, Leipzig, Germany.
}

\begin{abstract}
Myeloarchitecture of cerebral cortex has crucial implication on the function of cortical columnar modules. Based on the recent development of high-field magnetic resonance imaging (MRI), it was demonstrated that it is possible to individually reconstruct such intracortical microstructures. However, there is a scarcity of publicly available frameworks to perform group-wise statistical inferences on high resolution data. In this paper, we present a novel framework that parameterizes curved brain structures in order to construct correspondences across subjects without deforming individual geometry. We use the second Laplace-Beltrami eigenfunction to build such a parameterization, which is known to monotonically increase along the longest geodesic distance on an arbitrary manifold. To demonstrate our framework, a study on the lateralization of Heschl's gyrus is presented with multiple comparison correction.
\end{abstract}

Index Terms - Quantitative MRI, Myeloarchitecture, Cortical profile, Laplace-Beltrami operator, Shape parametrization, Heschl's gyrus, Lateralization

\section{INTRODUCTION}

The organization of neuronal cell bodies (cytoarchitecture) and myelinated fibers (myeloarchitecture) is an important basis for the quantification of columnar structures in cerebral cortex that are likely to function in a modular fashion. The cortical columns have often been characterized by cortical profiles that radially intersect the cortex in post-mortem slices, but recent development in high-field magnetic resonance imaging (MRI) has enabled in-vivo observation of such microstructures [1]. In particular, quantitative T1 mapping, which is known to inversely correlate with myelination, can be used to define myelination profiles.

Previous structural analyses usually incorporate a certain degree of deformation of the individual anatomy into a com-

${ }^{\star}$ The correspondence should be sent to S.-G.K (skim@cbs.mpg.de). This work is funded by International Max Planck Research School on Neuroscience of Communication. The authors would like to thank Jan Schreiber at MPI-CBS for discussion on segmentation tools.

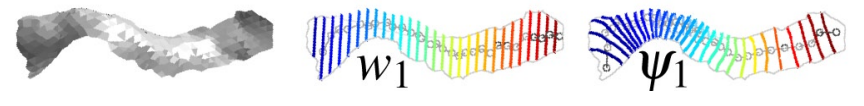

Fig. 1. For a given Heschl's gyrus (HG; left), isolevel contours of the first principal component $w_{1}$ in 3-D Euclidean space (middle) and the second Laplace-Beltrami eigenfunction $\psi_{1}$ (right) are shown. One can note that $w_{1}$ increases along a linear axis while $\psi_{1}$ increases naturally following the shape of HG (Section 2 for details).

mon space to establish intersubject correspondences in a 3D volume (e.g. voxel-based morphometry [2]) or on a 2-D surface (e.g. surface-based analysis [3]). However, it is unknown, to what extent, such distortion might introduce a spatial bias into statistical tests in the high-field MRI data.

Circumventing the registration issue of entire brains (see [4] for a spectral method for whole-brain matching), a spectral parametrization for a relatively well-defined brain region such as hippocampus or Heschl's gyrus (HG) could be a viable way to perform a statistical inference on group-wise differences based on theoretical hypotheses.

In this paper, we present a novel framework that parameterizes curved brain structures in order to construct correspondences across subjects without deforming individual geometries. For such parameterization, we propose to use the second eigenvector of the Laplace-Beltrami (LB) operator. The LB operator is a generalized Laplacian operator over an arbitrary manifold and its eigenfunctions have been used in biomedical imaging to extract intrinsic geometry of various structures $[5,6]$. Especially, the second LB eigenfunction is of interest for its monotonic property along the longest geodesic on the manifold [7]. This behavior was exploited to construct a curved medial axis of elongated structures such as the human mandible [6], the cingulate gyrus and the corpus callosum [8].

To demonstrate our proposed framework, a study on the lateralization of $\mathrm{HG}$, i.e. comparison between the left and right HGs will be explained in the following sections. Although HG has a relatively simple shape for a neuroanatomical structure, in reality, even HG often extends along a non- 
trivial axis. For instance, a spectrum in 3-D Euclidean space such as the first principle component shows its limitations in capturing the individual anatomy, as compared to the second LB eigenfunction in Figure 1. This illustrates our motivation to use LB eigenfunctions for parameterization. Statistical inferences will be also given testing some possible models with the application of multiple comparison correction.

\section{METHODS}

\subsection{Image acquisition and preprocessing}

Using a 7 T whole-body MR system (Siemens, Germany), two inversion images of six healthy subjects (all males; mean age $=25.7 \pm 2.2$ years) were acquired using the MP2RAGE sequence with an isotropic resolution of $0.7 \mathrm{~mm}$. T1-weighted images (T1w) and the longitudinal relaxation time $\mathrm{T}_{1}$ mapping (quantitative T1 map; qT1) were derived.

First voxels outside a head in T1w and qT1 images were masked by a thresholded second inversion image. Then nonbrain voxels were discarded using the segmentation tool in SPM8/VBM8 ${ }^{1}$. The images were aligned to the Montreal's Neurological Institute template (MNI152) using rigid-body transformation and up-sampled to $0.4 \mathrm{~mm}$ isovoxel, which is desirable to increase precision of layer extraction and cortical profile sampling (Section 2.3) since the particular computations are made with volumetric representations of surfaces as 3D signed distance maps.

\subsection{Surface reconstruction and manual delineation}

The outer boundary between gray matter and cerebrospinal fluid and the inner boundary between gray matter and white matter were semi-automatically constructed using FreeSurfer ${ }^{2}$. Due to the constraint of $1 \mathrm{~mm}$ resolution in the current public version of FreeSurfer, manual corrections were made with qT1 images in higher resolution after the initial surface generation. Reconstruction of cortical surfaces were iteratively done until the visual inspection on the region of interest (i.e. HG) was satisfactory.

From the final surface, a patch of superior temporal plane was cut and flattened. On the flattened patch, HGs were manually delineated based on criteria from literature [9] with alternative inspections on the original patch. The final segmentations of HGs were confirmed by a radiologist (A.V.). Examples of HG segmentations are shown in Figure 2. There were posterior duplication of $\mathrm{HG}$ and/or complete or incomplete sulcus intermedix either in both hemispheres $(n=4)$ or in one hemisphere $(n=2)$. For the sake of simplicity, the anterior gyrus, or the single $\mathrm{HG}$, is called $\mathrm{HG1}$ and the posterior gyrus, divided by the extension of SI, is called HG2 in the following.

\footnotetext{
${ }^{1}$ http://dbm.neuro.uni-jena.de/vbm/

${ }^{2}$ http://surfer.nmr.mgh.harvard.edu
}

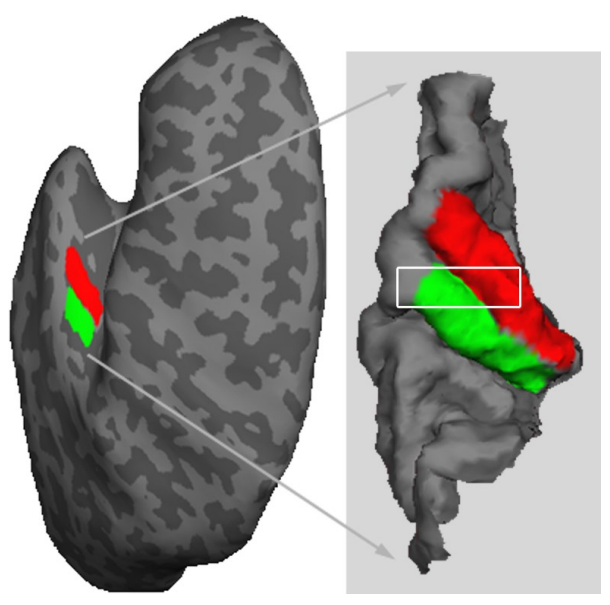

Fig. 2. The anterior HG (red) and the posterior duplication (green) on a left hemisphere.

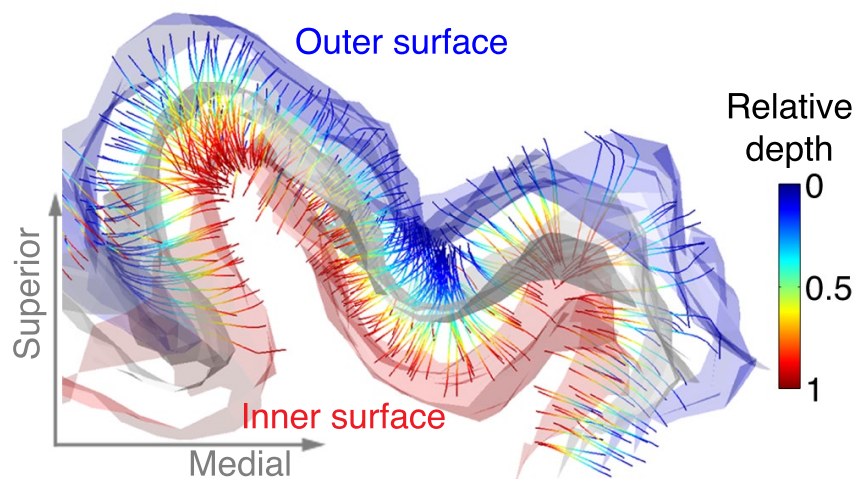

Fig. 3. For a region (white rectangle on the inset of Figure 2), constructed cortical profiles are visualized with the outer (blue), central (gray) and inner (red) surfaces.

\subsection{Realistic cortical layer estimation}

From the outer to inner surfaces, 19 intracortical layers were estimated using a realistic layer model [10], which is implemented in the high-field MRI processing tools [11] from our institute $^{3}$. This equi-volume model takes local curvatures into account and showed better compliance with a well-known striatal organization of primary cortices in $e x$ - and in-vivo data than Laplace- and equidistance-based models [10].

qT1 volumes at $0.4 \mathrm{~mm}$ isovoxel were directly sampled for each vertex of the central surface, which is an average of inner and outer surfaces. From the central surface, the vertices were first projected onto the nearest layer, then further projected onto both inward and outward layers until they reach the cortical boundaries. Due to the projection process, the equi-volumetric profiles do not necessarily connect the corresponding vertices of the inner and outer surfaces. Thus a myelination profile defined on a vertex is the characteristic of

\footnotetext{
${ }^{3}$ http://www.cbs.mpg.de/institute/software/cbs-hrt
} 

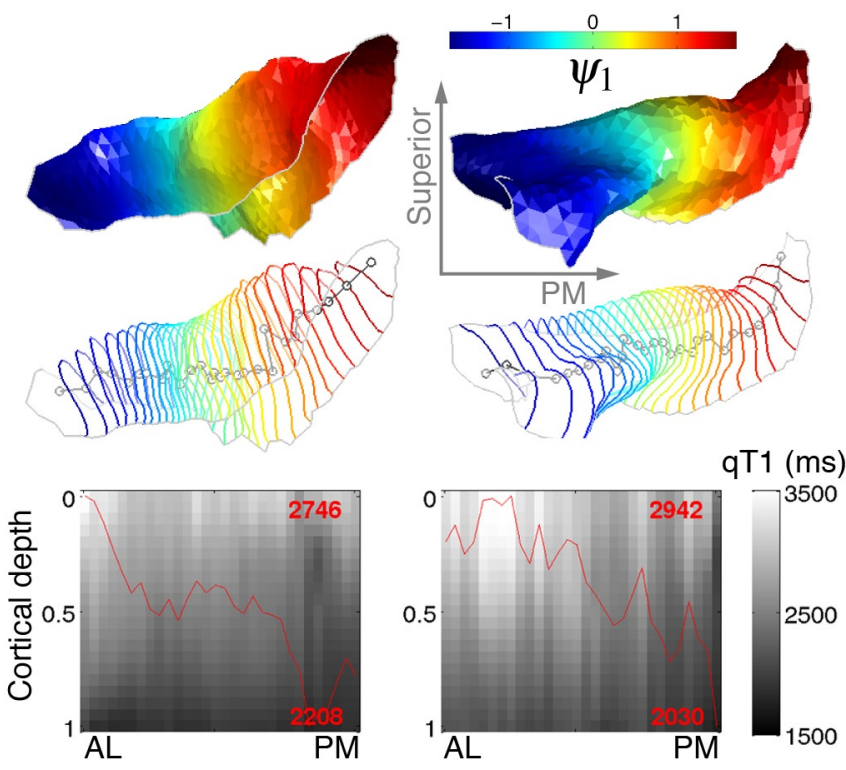

Fig. 4. Parametrization of myelination profiles based on the second LB eigenvector $\psi_{1}$ (upper) and discrete regions $\mathcal{R}(k)$ between isolevel contours (middle). The 2-D profile maps $\boldsymbol{I}$ (lower) are shown with superimposed plots (red) showing depth-wise average between the anterolateral (AL) and posteromedial (PM) endings.

the cortical profile that passes through the vertex. Estimated cortical profiles within the HGs of a subject are visualized in Figure 3.

\subsection{Laplace-Beltrami operator on discrete open mesh}

As mentioned earlier, we use the second LB eigenvector of HGs to build correspondence across subjects. Consider a Riemannian 2-manifold $\mathcal{M}$ embedded in $\mathbb{R}^{3}$. Eigenfunctions $\boldsymbol{\psi}_{j}$ of LB operator $\Delta$ defined on $\mathcal{M}$ satisfy

$$
\Delta \psi_{j}=-\lambda \psi_{j}
$$

and form orthonormal bases for a Hilbert space of square integrable functions that are defined on the manifold $\mathcal{M}$ [7]. As we order eigenvalues as $0=\lambda_{0}<\lambda_{1} \leq \lambda_{2} \leq \cdots$, the corresponding eigenfunctions are $\psi_{0}, \psi_{1}, \psi_{2} \cdots$.

Closed forms to solve the eigensystem Eq. (1) are only known for some manifolds such as a plane or a sphere [7]. In order to numerically compute Eq. (1) on an arbitrary manifold, we could discretize LB operator on a triangular mesh employing finite element method (FEM) [5, 7, 12]. The problem is now the following generalized eigenvalue problem:

$$
C \Psi=\lambda \boldsymbol{A} \Psi
$$

where $\boldsymbol{C}$ is cotangent matrix and $\boldsymbol{A}$ is mass matrix. Detailed explanations can be found in [7]. We used a MATLAB (Math- works, Natick, MA) implementation ${ }^{4}$ [13] that uses the algorithm given in [12] for meshes with any topology (either closed or open).

The second eigenvector $\psi_{1}$ was orientated such that the positive and negative peaks would be placed towards the posteromedial (PM) and anterolateral (AL) directions from the zero-valued center, respectively. Then we computed average myelination profiles of vertices $v$ that are included in the $k$-th region $\mathcal{R}(k)$ between adjacent levelsets of $\psi_{1}$ as

$$
\mathcal{R}(k)=\left\{v \in \mathcal{M} \mid \eta_{k} \leq \psi_{1}(v) \leq \eta_{k+1}\right\},
$$

where $\eta_{k}$ is the $k$-th contour level [8]. We set 30 regions from the AL to PM endings of the HGs. The mean distance between isolevel contours was $1.22 \pm 0.40 \mathrm{~mm}$ and the mean size of $\mathcal{R}(k)$ was $22.3 \pm 7.6$ vertices. The second LB eigenvectors of two HGs from different subjects and parametrized myelination profiles are given in Figure 4.

\subsection{Statistical inferences}

Once we parameterized the 2-D open patch into a 1-D representation, we can represent myelination profiles over $\mathrm{HG}$ as discrete 2-D images $\boldsymbol{I}$ with a dimension of 30 (AL - PM axis, or normalized $\psi_{1}$ ) by 21 (normalized cortical depth). As a demonstration of a group-wise analysis, here we test if there are any differences between the left and right HGs and/or between the first and second HGs. It can be inferred by fitting a general linear model (GLM) as

$$
d \boldsymbol{I}=\beta_{0}+\epsilon,
$$

with $d \boldsymbol{I}$ denoting a paired difference image of one subject while matching the laterality and the order of HGs. $\epsilon$ is Gaussian error with zero mean and unit standard deviation. An inference on a null hypothesis $H_{0}: \beta_{0}=0$ would be identical to the one-sample paired $t$-test.

We can also test the lateralization effect when we control the other variable with such a linear model as

$$
d \boldsymbol{I}=\beta_{0}+\beta_{1} X+\epsilon,
$$

where $X$ is a dummy covariate being either the order or the laterality of HGs. Note that our hypothesis is still $H_{0}: \beta_{0}=0$ since we are not interested in the term $\beta_{1} X$ here.

However, one can even test the interaction of the dummy variable with the paired differences by taking Eq. (5) as a full model and Eq. (4) as a reduced model into an F-test. Then the null hypothesis is $H_{0}: \beta_{1}=0$.

Since we test 630 pixels collectively that are highly dependent due to the smooth nature of biological signals and the partial volume effect, a MATLAB toolbox SurfStat ${ }^{5}$ was used for multiple comparison correction based on random field theory (RTF) [14]. The maps were minimally smoothed with the full width at half maximum of 2 edges to comply with the RTF assumption of the smoothness of field.

\footnotetext{
${ }^{4}$ http://www.di.ens.fr/ aubry/wks.html

${ }^{5}$ http://www.math.mcgill.ca/keith/surfstat/
} 


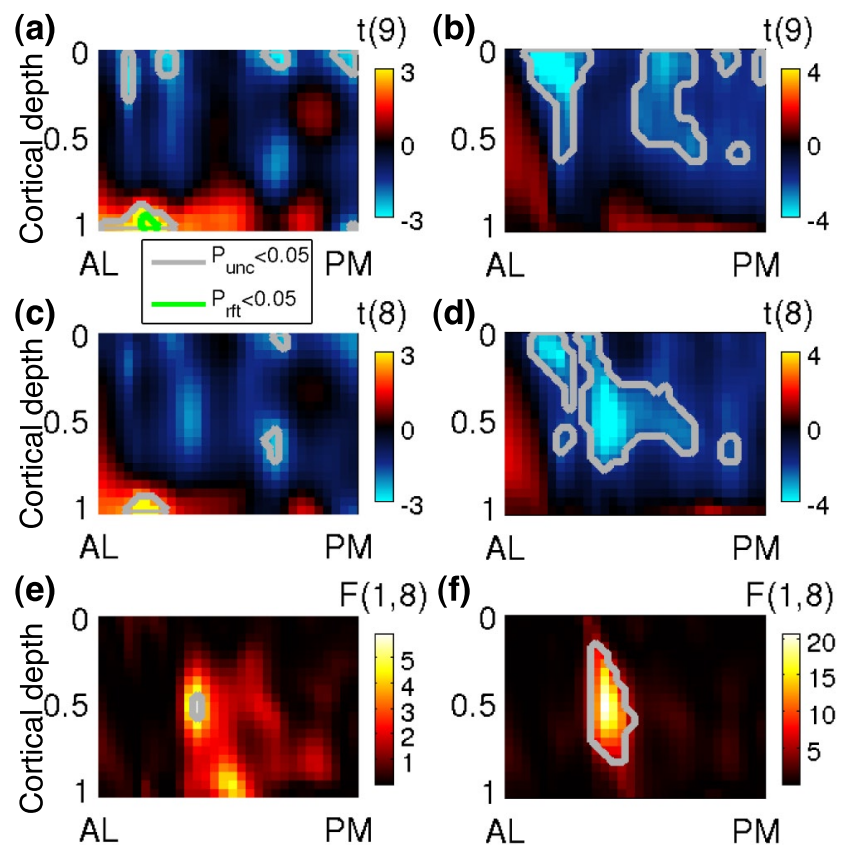

Fig. 5. Statistical inference on parameterized myelination profiles. GLM Eq. (4) (a,b), Eq. (5) (c,d) and interactions $(\mathbf{e , f})$ were tested for the effect of laterality $(\mathbf{a}, \mathbf{c}, \mathbf{e})$ and order $(\mathbf{b}, \mathbf{d}, \mathbf{f})$ of HGs. Contours mark the thresholds $(\alpha=0.05)$ in uncorrected $p$-values (gray) and corrected $p$-values (green).

\section{RESULTS AND DISCUSSION}

\subsection{Lateralization of $\mathbf{H G}$}

We found multiple signals for all models and contrasts (uncorrected $p<0.05$ ). However, after multiple comparison correction, the difference was significant only for the laterality tested by the simple model Eq. (4) $(\max t(9)=6.76$; min corrected $p=0.008$; Figure 5 (a)). It may be interpreted as that the myelination of the right $\mathrm{HG}$ was greater than the left $\mathrm{HG}$ given the inverse relationship between myelination and qT1 value. However, we cannot rule out the possibility of the partial volume effect as the signal lies near the inner boundary. In addition, the number of subjects is quite small. It should be noted that the primary purpose of the laterality study on HGs is to demonstrate our proposed group-wise framework. Even though the GLMs were given on the paired differences, one can easily adapt the given models simply by replacing the paired differences with response measurements.

\subsection{Extension of LB parameterization of HGs}

We have shown a group-wise analysis using the second LB eigenvector on the curved HGs. Although we simplified as HG1 and HG2 in the paper, the topological variability of HG sometimes involve even the higher degree of complexity. That is, not only the number of gyri but also the depth, length and locations of intermediate sulci differ across individuals and hemispheres. Utilizing the Reeb graph on LB eigenfunctions [8] might be helpful to deal with the topological variety in future works.

\section{REFERENCES}

[1] S. Geyer, M. Weiss, K. Reimann, G. Lohmann, and R. Turner, "Microstructural Parcellation of the Human Cerebral Cortex From Brodmann's Post-Mortem Map to in vivo Mapping with High-Field Magnetic Resonance Imaging," Front Hum Neurosci, vol. 5, no. February, pp. 19, 2011.

[2] J. Ashburner and K. Friston, "Voxel-based morphometry - the methods," NeuroImage, vol. 11, pp. 805-821, 2000.

[3] B. Fischl and A. M. Dale, "Measuring the thickness of the human cerebral cortex from magnetic resonance images," Proc Natl Acad Sci, vol. 97, no. 20, pp. 11050-11055, 2000.

[4] H. Lombaert, L. Grady, J. R Polimeni, and F. Cheriet, "Fast brain matching with spectral correspondence.," in Proc IPMI, 2011, vol. 22, pp. 660-673.

[5] A. Qiu, D. Bitouk, and M. I. Miller, "Smooth functional and structural maps on the neocortex via orthonormal bases of the Laplace-Beltrami operator," IEEE TMI, vol. 25, pp. 12961306, 2006.

[6] S. Seo, M. K. Chung, B. J. Whyms, and H. K. Vorperian, "Mandible shape modeling using the second eigenfunction of the Laplace-Beltrami operator," in Proc SPIE 7962, Med Img. SPIE, 2011, p. $79620 Z$.

[7] M. K. Chung, Computational Neuroanatomy: The Methods, World Scientific Publishing Company, 2012.

[8] Y. Shi, R. Lai, S. Krishna, N. Sicotte, I. Dinov, and A. W. Toga, "Anisotropic Laplace-Beltrami eigenmaps: Bridging Reeb graphs and skeletons," in Proc CVPRW. IEEE, 2008, pp. 1-7.

[9] P. Morosan, J. Rademacher, A. Schleicher, K. Amunts, T. Schormann, and K. Zilles, "Human primary auditory cortex: cytoarchitectonic subdivisions and mapping into a spatial reference system," NeuroImage, vol. 13, no. 4, pp. 684-701, 2001.

[10] M. D. Waehnert, J. Dinse, M. Weiss, M. N. Streicher, P. Waehnert, S. Geyer, R. Turner, and P.-L. Bazin, "Anatomically motivated modeling of cortical laminae," NeuroImage, 2013.

[11] P.-L. Bazin, M. Weiss, J. Dinse, A. Schäfer, R. Trampel, and R. Turner, "A computational framework for ultra-high resolution cortical segmentation at 7 Tesla," NeuroImage, 2013.

[12] M. Meyer, M. Desbrun, P. Schröder, and A. H. Barr, "Discrete differential-geometry operators for triangulated 2-manifolds," in Proc Visualization and Mathematics, 2002, pp. 1-26.

[13] M. Aubry, U. Schlickewei, and D. Cremers, "The wave kernel signature: A quantum mechanical approach to shape analysis," in Proc ICCV. IEEE, 2011, vol. 2, pp. 1626-1633.

[14] K. J. Worsley, J. E. Taylor, F. Carbonell, M. K. Chung, E. Duerden, B. Bernhardt, O. Lyttelton, M. Boucher, and A. C. Evans, "SurfStat: A Matlab toolbox for the statistical analysis of univariate and multivariate surface and volumetric data using linear mixed effects models and random field theory," NeuroImage, vol. 47, pp. S102-S102, 2009. 